\title{
SYNTHESIS AND BIOLOGICAL PROPERTIES OF PHARMACEUTICALLY IMPORTANT XANTHONES AND BENZOXANTHONE ANALOGS: A BRIEF REVIEW
}

\author{
POOJA BEDI, RICHA GUPTA, TANAY PRAMANIK* \\ Department of Chemistry, Faculty of Technology and Sciences, School of Chemical Engineering and Physical Science, Lovely Professional \\ University, Phagwara, Punjab, India. Email: Tanay.pramanik@gmail.com \\ Received: 06 September 2017, Revised and Accepted: 30 October 2017
}

\begin{abstract}
Xanthones are one of the biggest classes of compounds in natural product chemistry. A number of xanthones have been isolated from natural sources of higher plants such as fungi, ferns, and lichens. Synthetic analogs of xanthones have shown a large number of pharmacological properties such as antioxidant, anti-inflammatory, antidiabetics, antihistamine, antitumoral, antiulcer, and algicidal. Moreover, they also find usages in photodynamic therapy, laser technology, and dyes. This review lays stress on various solvents, catalyst and synthetic route for synthesis of xanthones, benzoxanthones analogs. The review has also focused on the classifications of xanthone as well as extensively studied biological properties of the xanthones and benzoxanthones analogs.
\end{abstract}

Keywords: Multicomponent reactions, Xanthones, Benzoxanthones, Biological properties.

(C) 2018 The Authors. Published by Innovare Academic Sciences Pvt Ltd. This is an open access article under the CC BY license (http://creativecommons. org/licenses/by/4. 0/) DOI: http://dx.doi.org/10.22159/ajpcr.2018.v11i2.22426

\section{INTRODUCTION}

During the past few decades, there has been widespread interest in multicomponent reactions (MCRs) due to their increasing importance in organic and medicinal chemistry [1-3]. MCRs are the processes in which three or more reactants are combined in a single step to yield products by combining suitable portions of all the reactants. These reactions are very effective in synthesizing highly functionalized small organic molecules from easily available starting materials in a single step and in short duration of time period. MCR also provides a higher overall percentage of yield. Thus these reactions reduces labor by reducing a number of synthetic operations such as extraction, purification, and generates lesser amount of waste as compare to conventional multistep reactions of a complex molecule [4-6]. A large number of MCRs are already known, and the searches for new MCRs are still on. One such reaction which belongs to MCR category is a synthesis of xanthones and its derivatives with extended conjugation, i.e., Benzoxanthones.

Xanthones are naturally occurring polyphenolic compounds. Xanthone nucleus is the main framework of large number of natural and synthetic materials. These are parent of several natural yellow pigments. These comprise an important class of oxygenated heterocycles. Xanthone Skelton possesses good thermal oxidative and hydrolytic stability, that's why these are considered as structural motif in high performance and engineering polymers [7]. Numerous derivatives of xanthones are isolated from higher plants, fungi, and lichens [8]. However, the naturally occurring xanthones are limited to a fewer number of substituent, so efforts are made to synthesize them from their constituent fragments.

Xanthones and benzoxanthones constitute an important class of biologically active heterocycles. Due to their remarkable pharmacological and biological applications, their synthesis has drawn great attention in the field of medicinal and pharmaceutical chemistry. They possess antiviral [9], anti-inflammatory [10], antibacterial [11], antimalarial [12], anti-HIV [13], antimicrobial, antioxidant, and anticarcinogenic $[14,15]$ activities. These are also used as an antagonist for paralyzing action of zoxazolamine [16]. Furthermore, these compounds can be used in photodynamic therapy [17], as dyes [18] in laser technology [19] and in fluorescent materials which are sensitive to $\mathrm{pH}$ for visualization of biomolecules [20].<smiles>O=c1c2ccccc2oc2ccccc12</smiles><smiles>CC1(C)CC(=O)C2=C(C1)Oc1ccc3ccccc3c1C2[14CH3]</smiles>

1. Xanthone

2. Benzoxanthone

\section{Classification of xanthone}

Naturally occurring xanthones are broadly classified into six categories:

1. Simple oxygenated xanthone.

2. Xanthone glycosides.

3. Prenylated xanthone.

4. Bisxanthones.

5. Xantholignoids.

6. Miscellaneous xanthones.

\section{Simple oxygenated xanthone}

These xanthones carry simple hydroxyl, methoxy, and methyl groups. These are further subdivided into various categories such as mono, $\mathrm{di}$, tri, tetra, penta, and hexa oxygenated depending on the level of oxygenation [21-23]. For example, 2-hydroxyxanthone (3), 2-hydroxy1-methoxyxanthone (4).

\section{Xanthone glycosides}

The xanthone in which sugar moiety is attached to xanthone nucleus is called xanthone glycosides. These are further of two types, i.e., C-glycosides and $\mathrm{O}$-glycosides. In C-glycosides sugar moiety is attached to xanthone nucleus by $\mathrm{C}-\mathrm{C}$ bond whereas in O-glycosides glycosidic linkage, i.e. C-O-C joins sugar moiety to xanthone. C-glycosides are fewer in number as compare to O-glycosides. For example, mangiferin (22) and isomangiferin are most common C-glycosides, and Swertia japonica (24) and gentioside are few 0-glycosides.

\section{Prenylated xanthone}

These are the xanthones in which 5-carbon unit such as isoprenyl and 1,1-dimethylprop-2-enyl,3-hydroxy-3-methylbutyl [24-26] is attached as a substituent to xanthone nucleus, for example, 


\section{Bisxanthones}

These are dimeric xanthones. First bisxanthone C-glycoside was swertipunicoside (26). It was isolated from Swertia punicea Hemsl plant. A few more examples are dimeric xanthone (27), globulixanthone E (28) [27], and ploiarixanthone. A total of 12 bisxanthones are known.

\section{Xantholignoids}

One of the most important category xanthone is xantholignoids. These were thought to be formed by coupling of cinnamoyl alcohol with an o-hydroxyxanthone. Some of the Xantholignoids are Kielcorin (29a) [28], 6-methylkielcorin (29b), Cadensin C (29c) [29], and Hypericorin (29d).

\section{Miscellaneous xanthone}

Xanthone with substituents other than those discussed above are included in this category. Few examples are xanthopterin [30], xantholiptin [31], and xanthofulvin (30) and vinaxanthone (31) [32].

\section{Synthesis of xanthone and benzoxanthone}

Synthesis of xanthone and its benzo-fused analogs, i.e., Benzoxanthone is extremely important due to its broad range of applications as mentioned above. Scientists have already invented an easy, efficient, and economic method to synthesize xanthone and benzoxanthone through one-pot multicomponent condensation reaction. Various schemes used by researchers to synthesize xanthone and benzoxanthone are mentioned here:

Scheme $1[33]$<smiles>Cc1ccccc1C(=O)c1cc(O)c(O)cc1O</smiles>

Scheme-1: a- CAN

water,chloroform,acetonitrile

Scheme $2[34]$

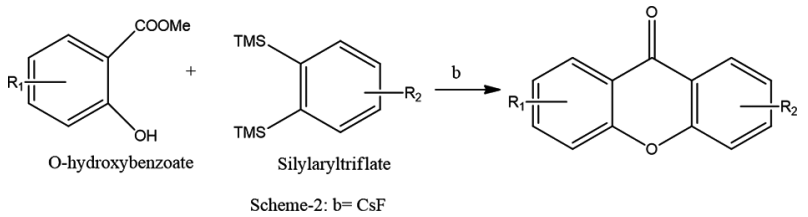

Scheme 3 [35]

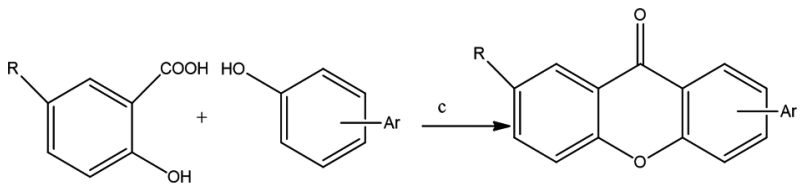

Scheme 3: $\mathrm{c}=\mathrm{POCl} 3, \mathrm{~b}=\mathrm{Heat}(80$ degree $\mathrm{C})$

Scheme 4 [36]

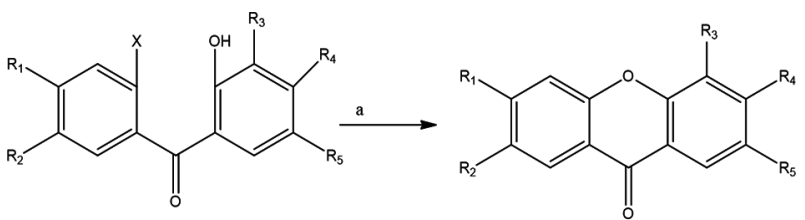
Scheme-1:a $=\mathrm{Cu}$, TMEDA water,Heat<smiles>[R]c1ccc2oc3ccc(O)cc3c(=O)c2c1</smiles>

Scheme-5: $\mathrm{a}=\mathrm{AlCl} 3 \quad \mathrm{~b}=\mathrm{Heat}, \mathrm{Cu}$

Scheme 6 [38]<smiles>[R9]C=CC1COc2ccccc2C1=O</smiles><smiles>COC(=O)c1cc2c(=O)c3ccccc3oc2c(Br)c1O</smiles>

Scheme 7 [38]<smiles>[R]C#CC1COc2ccccc2C1=O</smiles><smiles>NC(=O)CC(N)=O</smiles>
Scheme-7 a=DBU, $\mathrm{b}=\mathrm{DMF}, \mathrm{c}=\mathrm{RT}$<smiles>[R9]C(=O)c1cc2c(=O)c3ccccc3oc2c(Br)c1Br</smiles>

Scheme 8 [39]<smiles>[R]C#CC1C(=O)c2ccccc2OC1[R9]</smiles>

Scheme- $8 \mathrm{a}=\mathrm{DBU}, \mathrm{b}=\mathrm{DMF}, \mathrm{c}=\mathrm{MV}, \mathrm{d}=90$ degree $\mathrm{C}, \mathrm{d}=10 \mathrm{~min}$<smiles>[R3]c1ccc2oc3c([R2])c(N)c([R])c([R])c3c(=O)c2c1</smiles> 
Scheme $9[40]$<smiles>O=C1C=CC(=O)C=C1</smiles><smiles>COc1cccc(OC)c1C=O</smiles><smiles>O=C(O)O</smiles><smiles>COc1cccc2oc3ccc(O)cc3c(=O)c12</smiles>
Scheme- 9 a $=$ light, $b=\mathrm{KOH}, \mathrm{c}=\mathrm{BBr} 3$

Scheme 10 [41]<smiles>[R]c1cc2c(=O)c(C=O)coc2c([R3])c1[R]</smiles><smiles>COC(=O)OC(=O)OC(C)=O</smiles><smiles>[R]OC(=O)c1cc2c(=O)c3cc([R])cc(Br)c3oc2c(C(=O)O[Na])c1C(=O)O</smiles>

Scheme-10 : a=DME, DMAP, 4-Picoline,-18 degree C-RT

\section{Synthesis of benzoxanthone}

Scheme 1: [42] $\mathrm{a}=\mathrm{K}_{2} \mathrm{CO}_{3} / \mathrm{H}_{2} \mathrm{O}$

$\mathrm{b}=150^{\circ} \mathrm{C}, 6 \mathrm{~h}$<smiles></smiles>

Dihydroxybenzopenone

Dibe

Scheme 2: [43] a $=\mathrm{ZnCl}_{2}, \mathrm{~b}=\mathrm{POCl}_{3}$

$70-80^{\circ} \mathrm{C}, 1-5 \mathrm{~h}$<smiles></smiles>

Scheme 3: [43] a = $\mathrm{ZnCl}_{2}, \mathrm{~b}=\mathrm{POCl}_{3}$

$70-80^{\circ} \mathrm{C}, 1-5 \mathrm{~h}$<smiles>[R]c1cc([R])c2cc(O)c(C(=O)O)cc2c1</smiles><smiles>CC(C)(C)C</smiles><smiles>[R]c1cc([R])c2cc3oc4cc(O)cc(O)c4c(=O)c3cc2c1</smiles>

$\mathrm{R}_{1}=\mathrm{R}_{2}=\mathrm{H}$ Scheme -2

$\mathrm{R}_{1}=\mathrm{H}, \mathrm{R}_{2}=\mathrm{OH}$

Scheme 4: [44] a $=350 \mathrm{~nm}, \mathrm{~b}=\mathrm{MeOH}$<smiles>[R]c1ccc(C(=O)C(=O)c2ccc([R])cc2)cc1</smiles>

Scheme 5: [45] $\mathrm{a}=\mathrm{POCl}_{3}, 70^{\circ} \mathrm{C}$, heating $(6 \mathrm{~h})$<smiles>O=C(O)c1ccc2ccccc2c1O</smiles>

Scheme 6: [46] $\mathrm{a}=\mathrm{HBF}_{4} / \mathrm{SiO}_{2}, \mathrm{~b}=80^{\circ} \mathrm{C}$

or

$\mathrm{InCl}_{3}$ or $\mathrm{P}_{2} \mathrm{O}_{5}, 120^{\circ} \mathrm{C}$, solvent free [47]<smiles>CC1(C)CC(=O)CC(C)(C)C1</smiles><smiles></smiles>

Scheme 7A: [48] $\mathrm{a}=\mathrm{HClO}_{4} / \mathrm{SiO}_{2} \mathrm{~b}=80^{\circ} \mathrm{C}$ or 7B: [49] a $=\mathrm{SSA}, \mathrm{b}=80^{\circ} \mathrm{C}$, 15-210 min, solvent free or $7 \mathrm{C}$ : [50] $\mathrm{a}=\mathrm{H}_{2} \mathrm{SO}_{4}, \mathrm{~b}=\mathrm{H}_{2} \mathrm{O}$, reflux or 7D: [50] a $=$ PTS b $=$ MW, neat or 7E: [51] $\mathrm{HClO}_{4}, \mathrm{MW}$, solvent free 7F: [52] $\mathrm{a}=\mathrm{NaHSO}_{4} \cdot \mathrm{SiO}_{2}, \mathrm{~b}=\mathrm{CH}_{2} \mathrm{Cl}_{2}$ reflux $5 \mathrm{~h}$ or $7 \mathrm{G}$ : [53] a $=\mathrm{Sr}(\mathrm{OTf})_{2}$, $\mathrm{b}=1,2$-dichloroethane, $80^{\circ} \mathrm{C}$ or $7 \mathrm{H}$ : [54] PEG- 400 or 7I: [55] a $=\mathrm{ClSO}_{3} \mathrm{H}, \mathrm{b}$ = ultrasound irradiations or 7J: [56] $\mathrm{a}=\mathrm{TBAF}, \mathrm{b}=\mathrm{H}_{2} \mathrm{O}$ or $\mathrm{CH}_{2} \mathrm{Cl}_{2}$ or $\mathrm{CH}_{3} \mathrm{CN}$ or DMF or DMSO or $\mathrm{MeOH}, 100^{\circ} \mathrm{C} 7 \mathrm{~K}$ : [57] CAN, $120^{\circ} \mathrm{C}$, Solvent. free 


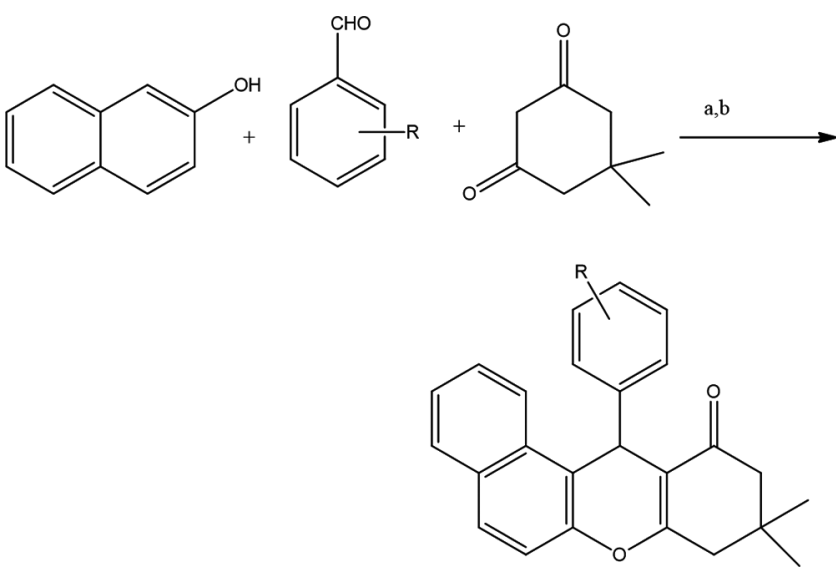

Scheme 8: [58] a = proline triflate, $\mathrm{b}=\mathrm{H}_{2} \mathrm{O}$<smiles>[R]c1c(N)c([R])c2oc3ccccc3c(=O)c2c1[R]</smiles>

11

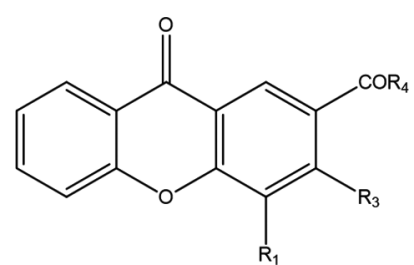

12<smiles>[R10]OC(=O)c1cc2c(c(C(=O)O[R20])c1C(=O)O[R12])Oc1c([R9])cc([R])cc1C2=C</smiles>

13<smiles>O=C1CCCC(=O)C1</smiles><smiles>Oc1cccc2ccccc12</smiles>
OR<smiles>[Y]C1C2=C(CCCC2=O)Oc2c1ccc1ccccc21</smiles><smiles>O=c1c2ccccc2oc2ccc(O)cc12</smiles><smiles>COc1c(O)ccc2oc3ccccc3c(=O)c12</smiles>

3<smiles></smiles><smiles>O=c1c2ccccc2oc2ccccc12</smiles>
$5\left(\mathrm{R}=\mathrm{CH}_{3}\right)$<smiles>O=c1c2cc(I)ccc2oc2ccc(I)cc12</smiles><smiles>[R9]C1=Cc2oc3cc([R9])c([R])cc3c(=O)c2C([R9])C1[R6]</smiles>

$7(\mathrm{R}=\mathrm{H}$ or $\mathrm{OH})$<smiles>O=c1c2cc(O)ccc2oc2ccc(Br)cc12</smiles>

8<smiles>O=c1c2cc(O)ccc2oc2cccc(O)c12</smiles>

10<smiles>[R1]#Cc1cccc2c(=O)c3cc(C(=O)OC)c(O)c(C)c3oc12</smiles>

14<smiles>O=c1c2c(ccc3ccc(O)cc32)oc2ccc3ccc(O)cc3c12</smiles>

15<smiles>[R]c1cc([R2])c2cc3oc4cc(O)cc(O)c4c(=O)c3cc2c1</smiles>

17<smiles>O=c1c2ccc(F)cc2oc2c3ccc(P)cc3c3cc(P)ccc3c12</smiles>

18<smiles>[R]C1C2=C(CCCC2=O)Oc2c1ccc1ccccc21</smiles>

20<smiles>O=c1c2c(O)cc(O)cc2oc2ccc3ccccc3c12</smiles>

16<smiles>[R]C1C2=C(CC(C)(C)CC2=O)Oc2ccc3ccccc3c21</smiles>

19<smiles>CC1(C)CC(=O)C2=C(C1)Oc1ccc3ccccc3c1C2c1ccccc1</smiles>

21 
<smiles>O=c1c2cc(O)c(O)cc2oc2cc(O)c(C3OC(CO)C(O)C(O)C3O)c(O)c12</smiles>

$22 \mathrm{R}=\mathrm{H}$

23 R=beta-D-glucopyranosyl<smiles></smiles>

24<smiles>C=CC(C)(C)c1c(O)c(CC=C(C)C)c(O)c2c(=O)c3cccc(O)c3oc12</smiles>

25<smiles>[Y9]c1c(O)c(-c2cc(O)c3oc4cc(O)cc(O)c4c(=O)c3c2O)c2oc3cc(O)c(O)cc3c(=O)c2c1O</smiles>

(c) $\mathrm{R} 1=\mathrm{H}, \mathrm{R} 2=$ olc-glc<smiles>CC(C)=CCc1ccc(-c2c(O)c(O)c(O)c3c(=O)c4c(O)cccc4oc23)c2c(=O)c3cc4c(cc3oc12)C=CC(C)(C)O4</smiles><smiles>O=c1c2c(O)cccc2oc2ccc(O)c(-c3c(O)ccc4oc5cccc(O)c5c(=O)c34)c12</smiles>

28

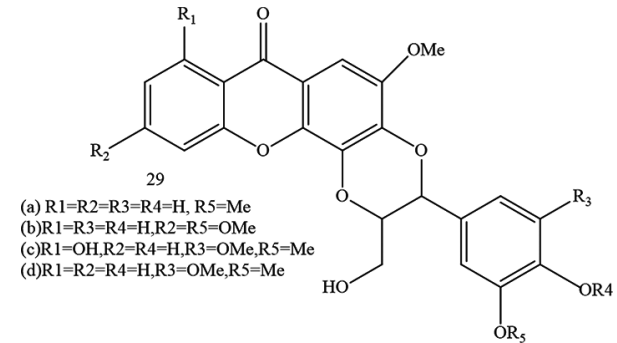<smiles>CC(=O)c1c(/C(O)=C2\COc3cc(O)c(O)c(C(=O)O)c3C2=O)cc2c(=O)c3c(C(=O)O)c(O)c(O)cc3oc2c1C(=O)O</smiles>

30<smiles>COc1c(-c2coc3cc(O)c(O)c(C(=O)O)c3c2=O)c(C(C)=O)cc2c(=O)c3c(C(=O)O)c(O)c(O)cc3oc12</smiles>

31<smiles>[R]c1c([R])c([R])c2c(=O)c3c([R])c([R])c([R])c([R])c3oc2c1[R]</smiles>

$32-50$<smiles>C=CC(C)(C)c1c2c(c(O)c3c(=O)c4cccc(O)c4oc13)C=CC(C)(C)O2</smiles>

51 
<smiles>[2H][C@H]1O[C@]2(C)c3c(cc(OC)c4c(=O)c5cccc(O)c5oc34)O[C@@]2(C)[C@H]1O</smiles><smiles>COc1cccc2c(=O)c3c(O)cc4c(c3oc12)[C@]1(C)OC[C@](O)(CO)[C@]1(C)O4</smiles><smiles>CC(C)(C)CCc1c(O)cc2c(=O)c3cc(O)c(O)c(CC(C)(C)C)c3oc2c1O</smiles><smiles>CC1(C)C=Cc2c(cc(Br)c3c(=O)c4ccccc4oc23)O1</smiles>
55<smiles>CC1(C)C=Cc2c(cc3oc4ccccc4c(=O)c3c2Br)O1</smiles>
56<smiles>Cc1ccc2c(=O)c3cccc(C(=O)O)c3oc2c1C</smiles>

57

Table 1: Various reaction condition, catalysts and solvents for synthesis of xanthones

\begin{tabular}{|c|c|c|c|c|c|}
\hline S. No. & Compound & Solvent & Catalyst & Reaction conditions & Reference \\
\hline 1 & 5 & $\mathrm{H} 2 \mathrm{O}$ or $\mathrm{CHCl} 3$ or $\mathrm{MeCN}$ & CAN & - & [33] \\
\hline 2 & 6 & $\begin{array}{l}\text { Various solvents like } \mathrm{CH} 3 \mathrm{COCH} 3, \mathrm{CH} 3 \mathrm{CN}, \mathrm{C} 6 \mathrm{H} 5 \mathrm{CH} 3 \mathrm{THF} \text {, } \\
\text { DME, } \mathrm{CH}_{3} \mathrm{NO} 2, \mathrm{CH} 2 \mathrm{Cl} 2\end{array}$ & $\mathrm{CsF}$ & $\mathrm{RT}-100^{\circ} \mathrm{C}$ & [34] \\
\hline 3 & 7 & - & POCl3 & Heat $\left(80^{\circ} \mathrm{C}\right)$ & [35] \\
\hline 4 & 8 & $\mathrm{H} 2 \mathrm{O}$ & $\mathrm{Cu}$, TMEDA & Heat & [36] \\
\hline 5 & 9 & - & $\mathrm{AlCl} 3$ & Heat & [37] \\
\hline 6 & 10 & Boiling $\mathrm{CH} 3 \mathrm{OH}$ & $\mathrm{KOH}$ & Light & [40] \\
\hline 7 & 11 & - & - & $\mathrm{DBU} / \mathrm{DMF}, \mathrm{MV}, 90^{\circ} \mathrm{C}, 10 \mathrm{~min}$ & [39] \\
\hline 8 & 12 & - & - & DBU/DMF, RT & [38] \\
\hline 9 & 13 & DME & 4-Picoline, MAP & Heat $\left(-18^{\circ} \mathrm{C}-\mathrm{RT}\right)$ & [41] \\
\hline 10 & 14 & - & - & $\mathrm{DBU} / \mathrm{DMF}, 45^{\circ} \mathrm{C}$ & {$[38]$} \\
\hline
\end{tabular}

Table 2: Various reaction condition, catalysts, and solvents for synthesis of benzoxanthones

\begin{tabular}{|c|c|c|c|c|c|}
\hline S. No. & Compound & Solvent & Catalyst & Reaction conditions & Reference \\
\hline 1 & 15 & $\mathrm{H}_{2} \mathrm{O}$ & $\mathrm{K}_{2} \mathrm{CO}_{3}$ & Heat $\left(150^{\circ} \mathrm{C}\right), 6 \mathrm{~h}$ & [42] \\
\hline \multirow[t]{2}{*}{2} & 16 & -2 & $\mathrm{ZnCl}_{2}^{2}, \mathrm{POCl}_{3}$ & Heat $\left(70-80^{\circ} \mathrm{C}\right), 1-5 \mathrm{~h}$ & [43] \\
\hline & & - & $\mathrm{POCl}_{3}^{2} \quad{ }^{3}$ & Heat $\left(70^{\circ} \mathrm{C}\right), 6 \mathrm{~h}$ & [45] \\
\hline 3 & 17 & - & $\mathrm{ZnCl}_{2}^{3}, \mathrm{POCl}_{3}$ & Heat $\left(70-80^{\circ} \mathrm{C}\right), 1-5 \mathrm{~h}$ & [43] \\
\hline 4 & 18 & $\mathrm{CH}_{3} \mathrm{OH}$ & - & Light radiations (350 nm) & [44] \\
\hline \multirow[t]{3}{*}{5} & 19 & $-{ }^{3}$ & $\mathrm{HBF}_{4}-\mathrm{SiO}_{2}$ & Heat $\left(80^{\circ} \mathrm{C}\right)$ & [46] \\
\hline & & - & $\mathrm{InCl}_{3}$ or $\mathrm{P}_{2} \mathrm{O}_{5}$ & Heat $\left(120^{\circ} \mathrm{C}\right)$ & [47] \\
\hline & & $\mathrm{H}_{2} \mathrm{O}$ & Proline triflate & - & [58] \\
\hline 6 & 20 & $\mathrm{H}_{2}^{2} \mathrm{O}$ & Proline triflate & - & [58] \\
\hline \multirow[t]{11}{*}{7} & 21 & - & $\mathrm{HClO}_{4}-\mathrm{SiO}_{2}$ & Heat $\left(80^{\circ} \mathrm{C}\right)$ & [48] \\
\hline & & - & SSA ${ }^{4}$ & Heat $(80 \mathrm{C}), 15-210 \mathrm{~min}$ & [49] \\
\hline & & $\mathrm{H}_{2} \mathrm{O}$ & $\mathrm{H}_{2} \mathrm{SO}_{4}$ & Reflux $(3 \mathrm{~h})$ & [50] \\
\hline & & $-{ }^{2}$ & PTS & MW & [50] \\
\hline & & - & $\mathrm{HClO}_{4}$ & MW & [51] \\
\hline & & DCM & $\mathrm{NaHSO}_{4}-\mathrm{SiO}_{2}$ & Reflux (5 h) & [52] \\
\hline & & 1,2-DCE & $\operatorname{Sr}(\mathrm{OTf})$ & Heat $\left(80^{\circ} \mathrm{C}\right)$ & [53] \\
\hline & & PEG-400 & & Heat $120^{\circ} \mathrm{C}(5-8 \mathrm{~h})$ & [54] \\
\hline & & - & $\mathrm{ClSO}_{3} \mathrm{H}$ & Ultrasound & [55] \\
\hline & & $\mathrm{H}_{2} \mathrm{O}$ or DCM or $\mathrm{MeCN}$ or $\mathrm{MeOH}$ or THF or DMSO or DMF & TBAF & Heat $\left(100^{\circ} \mathrm{C}\right)$ & [56] \\
\hline & & $-{ }^{2}$ & CAN & Heat $120^{\circ} \mathrm{C}$ & [57] \\
\hline
\end{tabular}


Table 3: Biological properties of xanthones and their analogs

\begin{tabular}{|c|c|c|c|c|}
\hline S. No. & Biological property & Compound & Compound name & Reference \\
\hline 1 & Antimalarial & 32 & $2,3,4,5,6$-pentahydroxyxanthone (X5) $\mathrm{R}^{2}=\mathrm{R}^{3}=\mathrm{R}^{4}=\mathrm{R}^{5}=\mathrm{R}^{6}=\mathrm{OH}, \mathrm{R}^{1}=\mathrm{R}^{7}=\mathrm{R}^{8}=\mathrm{H}$ & [12] \\
\hline \multirow[t]{3}{*}{2} & Antimicrobial & 51 & Inoxanthone & [59] \\
\hline & & 52 & Psorofebrin & {$[60]$} \\
\hline & & 53 & 5/-Hydroxyisopsorofebrin & \\
\hline \multirow[t]{2}{*}{3} & Antidiabetic & 22 & Mangiferin (xanthone glucoside) & [61] \\
\hline & & 23 & Mangiferin-7-0-beta-gluoside & \\
\hline \multirow[t]{2}{*}{4} & Antiplatelet aggregation & 33,34 & $\begin{array}{l}2,3,6,7\left(R^{2}=R^{3}=R^{6}=R^{7}=0 A c, R^{1}=R^{4}=R^{5}=R^{8}=H\right) \text { and } 3,4,6,7\left(R^{3}=R^{4}=R^{6}=R^{7}=0 A c\right. \\
\left.R^{1}=R^{2}=R^{5}=R^{8}=H\right) \text { tetrahydroxyxanthone tetraacetate }\end{array}$ & {$[62]$} \\
\hline & & 35 & $1,3,5,6$-tetrahydroxyxanthone $\mathrm{R}^{1}=\mathrm{R}^{3}=\mathrm{R}^{5}=\mathrm{R}^{6}=\mathrm{OH}, \mathrm{R}^{2}=\mathrm{R}^{4}=\mathrm{R}^{7}=\mathrm{R}^{8}=\mathrm{H}$ & \\
\hline \multirow[t]{2}{*}{5} & Antiviral & 22 & Mangiferin (xanthone glucoside) & [63] \\
\hline & & 54 & Mangostin $(\mathrm{R}=\mathrm{CH} 3)$ Gamma mangostin $(\mathrm{R}=\mathrm{H})$ & {$[64]$} \\
\hline \multirow[t]{2}{*}{6} & Antioxidant & & $\begin{array}{l}\text { Garcinia indica extract G. Mangostana extract G. Kola extract G. Atroviridis crude } \\
\text { extract }\end{array}$ & [65-68] \\
\hline & & 36,37 & $\begin{array}{l}\text { 2,3,6,8-tetrahydroxy-1-methylxanthone } \mathrm{R}^{2}=\mathrm{R}^{3}=\mathrm{R}^{6}=\mathrm{R}^{8}=\mathrm{OH}, \mathrm{R}^{4}=\mathrm{R}^{5}=\mathrm{R}^{7}=\mathrm{H}, \mathrm{R}^{1}=\mathrm{Me} \\
\text { and 3,6,8-trihydroxy-1-methylxanthone } \mathrm{R}^{3}=\mathrm{R}^{6}=\mathrm{R}^{8}=\mathrm{OH}, \mathrm{R}^{2}=\mathrm{R}^{4}=\mathrm{R}^{5}=\mathrm{R}^{7}=\mathrm{H} \mathrm{R}^{1}=\mathrm{Me}\end{array}$ & [69] \\
\hline \multirow[t]{3}{*}{7} & Antifungal & & Garcinia indica extract or G. atroviridis crude extract & {$[65,68]$} \\
\hline & & 38 & 1,7-dihydroxy-4-methoxyxanthone $\left(\mathrm{R}^{1}=\mathrm{R}^{7}=\mathrm{OH}, \mathrm{R}^{2}=\mathrm{R}^{3}=\mathrm{R}^{5}=\mathrm{R}^{6}=\mathrm{R}^{8}=\mathrm{H}, \mathrm{R}^{4}=\mathrm{OMe}\right)$ & [70] \\
\hline & & 39 & 1,7-dihydroxy-3,5,6-trimethoxyxanthone $\left(\mathrm{R}^{1}=\mathrm{R}^{7}=\mathrm{OH}, \mathrm{R}^{3}=\mathrm{R}^{5}=\mathrm{R}^{6}=\mathrm{OMe}, \mathrm{R}^{2}=\mathrm{R}^{4}=\mathrm{R}^{8}=\mathrm{H}\right.$ & \\
\hline \multirow[t]{2}{*}{8} & Antiasthmatic & 40 & $\begin{array}{l}\text { 7-methylsulfinylxanthone-2-carboxylic Acid }\left(\mathrm{R}^{7}=\mathrm{SOMe}^{2}=\mathrm{COOX}(\mathrm{X}=\mathrm{H} \text { or } \mathrm{Na}) \text {, }\right. \\
\left.\mathrm{R}^{1}=\mathrm{R}^{3}=\mathrm{R}^{4}=\mathrm{R}^{5}=\mathrm{R}^{6}=\mathrm{R}^{8}=\mathrm{H}\right)\end{array}$ & [71] \\
\hline & & 41 & $\begin{array}{l}\text { 7- }\left(\text { methylthio) xanthone-2-carboxylic acid }\left(\mathrm{R}^{7}=\mathrm{SMe}, \mathrm{R}^{2}=\mathrm{COOX}(\mathrm{X}=\mathrm{H} \text { or } \mathrm{Na}) \text {, }\right.\right. \\
\left.\mathrm{R}^{1}=\mathrm{R}^{3}=\mathrm{R}^{4}=\mathrm{R}^{5}=\mathrm{R}^{6}=\mathrm{R}^{8}=\mathrm{H}\right)\end{array}$ & \\
\hline 9 & Analgesic & & Mangifera extract & [74] \\
\hline 10 & Antitumoral & & Garcinia atroviridis extract & [68] \\
\hline \multirow[t]{3}{*}{11} & Anticancer & 42 & (a) Oxygenated xanthones $\left(\mathrm{R}^{2}=\mathrm{R}^{4}=\mathrm{R}^{5}=\mathrm{R}^{6}=\mathrm{R}^{7}=\mathrm{R}^{8}=\mathrm{OH} / \mathrm{Me} / \mathrm{OMe} / \mathrm{CHO}\right)$ & [75] \\
\hline & & 55,56 & Pyranoxanthenone $\left(\mathrm{R}=\mathrm{CONHCH}_{2} \mathrm{CH}_{2} \mathrm{NR}^{\prime} \mathrm{R}^{\prime}\right.$ or $\mathrm{CH}_{2} \mathrm{NHCH}_{2} \mathrm{CH}_{2} \mathrm{NR}^{\prime} \mathrm{R}^{\prime}$ & [76] \\
\hline & & 57 & DMXXA (Dimethylxanthone-4- acetic acid) & [77] \\
\hline \multirow[t]{10}{*}{12} & Anti-inflammatory & & (a) Garcinia mangostana extract & {$[66,67,72,74]$} \\
\hline & & & $\begin{array}{l}\text { (b) G. Kola extract } \\
\text { (c) Vimang (aqueous extract of Mangifera indica) }\end{array}$ & \\
\hline & & $43-50$ & (a) 1,3-dihydroxyxanthone $\left(\mathrm{R}_{1}=\mathrm{R}_{3}=\mathrm{OH}\right)$ & [73] \\
\hline & & & (b)3,5-dihydroxyxanthone $\left(\mathrm{R}_{3}=\mathrm{R}_{5}=\mathrm{OH}\right)$ & \\
\hline & & & (c)1,6-dihydroxyxanthone $\left(\mathrm{R}_{1}=\mathrm{R}_{6}=\mathrm{OH}\right)$ & \\
\hline & & & (d)1,3,7-trihydroxyanthone $\left(\mathrm{R}_{1}=\mathrm{R}_{3}=\mathrm{R}_{7}=\mathrm{OH}\right)$ & \\
\hline & & & (e)1,3,8-trihydroxyanthone $\left(\mathrm{R}_{1}=\mathrm{R}_{3}=\mathrm{R}_{8}=\mathrm{OH}\right)$ & \\
\hline & & & (f) 1,3,5,6-terahydroxyxanthone $\left(\mathrm{R}_{1}=\mathrm{R}_{3}=\mathrm{R}_{5}=\mathrm{R}_{6}=\mathrm{OH}\right)$ & \\
\hline & & & (g)2,3,6,7-terahydroxyxanthone $\left(\mathrm{R}_{2}=\mathrm{R}_{3}=\mathrm{R}_{6}=\mathrm{R}_{7}=\mathrm{OH}\right)$ & \\
\hline & & & (h) 3,4,5,6-terahydroxyxanthone $\left(\mathrm{R}_{3}=\mathrm{R}_{3}=\mathrm{R}_{5}=\mathrm{R}_{6}=\mathrm{OH}\right)$ & \\
\hline
\end{tabular}

From Table 1, it is evident that xanthones can be synthesized using various solvents under harsh reaction conditions. Most of the solvents employed for the preparation of xanthone are not environmentally, and the catalysts used for xanthones synthesis are not ecofriendly. Most effective method is the one which is carried out under solvent-free and catalyst-free conditions at room temperature or in microwave (for compounds 11,12, and 14).

Table 3, for synthesis of benzoxanthone, shows that they can be prepared by employing a number of solvents and also under neat conditions by making use of variety of acidic catalysts under different reaction conditions such as heat, microwave, ultrasound, and visible radiations. However, use of microwave, ultrasound and visible radiations is preferred to carry out reaction they are considered as green methodology.

Table 3 summarizes a list of almost all the literature reported biological properties of xanthones and their derivatives. These compounds are used for the treatment of a number of diseases such as diabetes, cancer, malaria and also the diseases caused by herpes virus, bacteria, and fungi. Extracts of plants containing xanthone and their analogs are also employed for curing allergic, inflammatory, cardiotonic, convulsant, mutagenic, analgesic, ulcergenic, etc., activities. Due to their remarkable pharmacological and biological activities, it has now become an essential part of chemistry to study their synthesis.

\section{CONCLUSION}

This review summarizes not only various synthetic routes for the synthesis of xanthones and benzoxanthones but also the pharmaceutical and biological significance of these compounds in different area have been highlighted in this review.

\section{AUTHORS CONTRIBUTION}

All the authors have contributed equally.

\section{CONFLICT OF INTEREST}

Declared none.

\section{REFERENCES}

1. Armstrong RW, Combs AP, Tempest PA, Brown SD, Keating TA. Multiple-component condensation strategies for combinatorial library synthesis. Acc Chem Res 1996;29:123-31.

2. a) Tietze LF, Lieb ME. Domino reactions for library synthesis of small molecules in combinatorial chemistry. Curr Opin Chem Biol 1998;2:363-71. b) Pramanik T, Padan SK. Microwave irradiated "green biginelli reaction" employing apple, pomegranate and grape juice as eco-friendly reaction medium. Int J Pharm Pharm Sci 2016;8:396-8. c) Pramanik T, Maji P. Microwave assisted green synthesis of pharmaceutically importantdihydropyrimidinones in fruit juice medium. Int J Pharm Pharm Sci 2015;7:376-9. 
3. Dax SL, McNally JJ, Youngman MA. Multi-component methodologies in solid-phase organic synthesis. Curr Med Chem 1999;6:255-70.

4. Plunkett MJ, Ellman JA. Combinatorial chemistry and new drugs. Sci Am 1997;276:68-73

5. Schreiber SL. Target-oriented and diversity-oriented organic synthesis in drug discovery. Science 2000;287:1964-9.

6. Kappe CO. Recent advances in the biginelli dihydropyrimidine synthesis. New tricks from an old dog. Acc Chem Res 2000;33:879-88.

7. Colquhoun HM, Lewis DF, Williams DJ. Synthesis of dixanthones and poly(dixanthone)s by cyclization of 2-aryloxybenzonitriles in trifluoromethanesulfonic acid. Org Lett 2001;3:2337-40.

8. Cardona ML, Fernandez MI, Pedro JR, Serrano A. Xanthones from Hypericum reflexum. Phytochemistry 1990;29:3003-6.

9. Zheng MS. Antiviral effect of mangiferin and isomangiferin on herpes simplex virus. Chin Med J 1990;103:160-5.

10. a) Poupelin JP, Saint-Ruf G, Foussard-Blanpin O, Narcisse G, UchidaErnouf G, Lacroix R. Synthesis and anti-inflammatory properties of bis(2-hydroxy, 1-naphthyl) methane derivatives. Eur J Med Chem 1978;13:67-71. b) Mahendran G, Manoj M, Prasad KJ, Bai VN. Evaluation of anti-inflammatory and antinoceceptive activity of xanthones from Swertia corymbosa (Griseb.) Wight ex c.b. Clarke. Int J Pharm Pharm Sci 2013;5:523-9.

11. Mari S, Rossi M, Valenti P, Da Re P. Flavone and xanthone derivatives related to fluoroquinolones. Farmaco 1999;54:411-5.

12. Ignatushchenko MV, Winter RW, Bächinger HP, Hinrichs DJ, Riscoe MK. Xanthones as antimalarial agents; studies of a possible mode of action. FEBS Lett 1997;409:67-73

13. Groweiss A, Cardellina JH, Boyd MR. HIV-Inhibitory prenylated xanthones and flavones from Maclura tinctoria. J Nat Prod 2000;63:1537-9.

14. a) Chen YL, Chen PH, Chung $\mathrm{CH}$, Li KC, Jeng HY, Tzeng CC, et al. Synthesis and cytotoxicity evaluation of metal-chelator-bearing flavone, carbazole, dibenzofuran, xanthone, and anthraquinone. Helvetica 2003;86:778-86. b) Ngoupayo J, Tabopda TK, Ali MS. Antimicrobial and immunomodulatory properties of Prenylated xanthones from twigs of Garcinia staudtii. Bioorg Med Chem 2009; 17:5688-95

15. a) Zhao Y, Liu JP, Lu D, Li PY, Zhang LX. A new antioxidant xanthone from the pericarp of Garcinia mangostana Linn. Nat Prod Res 2010;24:1664-70. b) Luo CT, Mao SS, Liu FL, Yang MX, Chen H, Kurihara H, et al. Antioxidant xanthones from Swertia mussotii, a high altitude plant. Fitoterapia 2013;91:140-7.

16. Saint-Ruf G, Huynh-Trong-Hieu, Poupelin JP. The effect of dibenzoxanthenes on the paralyzing action of zoxazolamine. Naturwissenschaften 1975;62:584-5.

17. Ion RM, Planner A, Wiktorowicz K, Frackowiak D. The incorporation of various porphyrins into blood cells measured via flow cytometry, absorption and emission spectroscopy. Acta Biochim Pol 1998;45:833-45

18. Banerjee A, Mukherjee AK. Chemical aspects of santalin as a histological stain. Stain Technol 1981;56:83-5.

19. Ahmad M, King TA, Ko DK, Cha BH, Lee J. Performance and photostability of xanthene and pyrromethene laser dyes in sol-gel phases. J Phys D Appl Phys 2002;35:1473-6.

20. Knight CG, Stephens T. Xanthene-dye-labelled phosphatidy lethanolamines as probes of interfacial $\mathrm{pH}$. Studies in phospholipid vesicles. Biochem J 1989;258:683-7.

21. Vieira LM, Kijjoa A. Naturally-occurring xanthones: Recent developments. Curr Med Chem 2005;12:2413-46

22. Mandal S, Das PC, Joshi PC. Naturally-occurring xanthones from terrestrial flora. J Indian Chem Soc 1992;69:611-36.

23. Sultanbawa MU. Xanthonoids of tropical plants. Tetrahedron 1980;36:1465-6.

24. Bringmann G, Lang G, Steffens S, Günther E, Schaumann K. Evariquinone, isoemericellin, and stromemycin from a sponge derived strain of the fungus Emericella variecolor. Phytochemistry 2003;63:437-43

25. Nguyen LH, Harrison LJ. Xanthones and triterpenoids from the bark of Garcinia vilersiana. Phytochemistry 2000;53:111-4.

26. Rukachaisirikul V, Kamkaew M, Sukavisit D, Phongpaichit S, Sawangchote P, Taylor WC. Antibacterial xanthones from the leaves of Garcinia nigrolineata. J Nat Prod 2003;66:1531-5.

27. Nkengfack AE, Mkounga P, Meyer M, Fomum ZT, Bodo B. Globulixanthones C, D and E: Three prenylated xanthones with antimicrobial properties from the root bark of Symphonia globulifera. Phytochemistry 2002;61:181-7.
28. Nielsen H, Arends P. Structure of the xanthonolignoid kielcorin. Phytochemistry 1978;17:2040-1.

29. Nikolaeva GG, Glyzin VI, Mladentseva MS, Sheichenko VI, Patudin AV. Xanthones of Gentiana lutea. Chem Nat Comp 1983;19:106-7.

30. Terui Y, Yiwen C, Jun-Ying L. Xantholiptin, a novel inhibitor of HSP47 gene expression produced by Streptomyces sp, Tetra. Letters 2003;44:5427-30.

31. Yang C, Li MA, Zhen-Ping WE, Feng HA, Jing GA. Advances in isolation and synthesis of xanthone derivatives. Chin Her Med 2012;4:887-902.

32. Kumagai K, Hosotani N, Kikuchi K, Kimura T, Saji I. Xanthofulvin, a novel semaphorin inhibitor produced by a strain of Penicillium. J Antibiot (Tokyo) 2003;56:610-6.

33. Johnson MM, Naidoo JM, Fernandes MA, Mmutlane EM, van Otterlo WA, de Koning CB, et al. CAN-mediated oxidations for the synthesis of xanthones and related products. J Org Chem 2010;75:8701-4.

34. Zhao J, Larock RC. Synthesis of xanthones, thioxanthones, and acridones by the coupling of arynes and substituted benzoates. J Org Chem 2007;72:583-8.

35. Lakouraj MM, Tashakkorian H, Rouhi M. One-pot synthesis of xanthones and dixanthones using calix[4]arene sulfonic acid under solvent free condition. Chem Sci Trans 2013;2:739-48.

36. Barbero N, Sanmartin R, Daminguez E. An efficient copper-catalytic system for performing intramolecular $\mathrm{O}$-arylation reactions in aqueous media. New synthesis of xanthones. Green Chem 2009;11:830-6.

37. Oleinik AF, Adamskaya EV. 3-Aryl- and 3-(aryloxy)phthalic acids in the synthesis of fluorenones and xanthones. Chem Heterocycl Comp 2007; 19:1221-4

38. Zhao L, Xie F, Cheng G, Hu Y. A base-promoted tandem reaction of 3-(1-alkynyl)chromones with 1,3-dicarbonyl compounds: An efficient approach to functional xanthones. Angew Chem Int Ed Engl 2009;48:6520-3

39. Liu Y, Huang L, Xie F, Hu Y. Base-promoted one-pot tandem reaction of 3-(1-alkynyl)chromones under microwave irradiation to functionalized amino-substituted xanthones. J Org Chem 2010;75:6304-7.

40. Kraus GA, Mengwasser J. Quinones as key intermediates in natural products synthesis. Syntheses of bioactive xanthones from Hypericum perforatum. Molecules 2009; 14:2857-61.

41. Terzidis MA, Tsiaras VG, Stephanidou-Stephantou J, Tsoleridis CA. Organocatalyzed reactions involving 3-formylchromones and acetylenedicarboxylates: Efficient synthesis of functionalized benzophenones and polysubstituted xanthones. Synthesis 2011;1:897-903

42. Azuma E, Kuramochi K, Tsubaki K. Alternative simple and effective synthesis of (di)benzoxanthones and their functions toward fluorescent dyes. Tetrahedron 2013;69:1694-9.

43. Liu Y, Ma L, Chen WH, Wang B, Xu ZL. Synthesis of xanthone derivatives with extended pi-systems as alpha-glucosidase inhibitors: Insight into the probable binding mode. Bioorg Med Chem 2007; 15:2810-4.

44. Kim SS, Lin CH, Yoo DY, Joong K, Ahn BJ, Shim SC. One pot preparation of xanthone derivatives from aromatic $\alpha$-diketones. Bull Korean Chem Soc 1993;14:661-3.

45. Cheng P, Zhu L, Guo W, Liu W, Yao J, Dong G, et al. Synthesis of novel benzoxanthone analogues as non-Camptothecin topoisomerase I inhibitors. J Enzyme Inhib Med Chem 2012;27:437-42.

46. Zhang ZH, Wang HJ, Ren XQ. A facile and efficient method for synthesis of xanthone derivatives catalyzed by $\mathrm{HBF} 4 / \mathrm{SiO} 2$ under solvent-free conditions. Monatshr Chem 2009;140:1481-3.

47. Nandi GC, Samai S, Kumar R, Singh MS. An efficient one-pot synthesis of tetrahydrobenzo[a]xanthene-11-one and diazabenzo[a]anthracene9,11-dione derivatives under solvent free condition. Tetrahedron 2009;65:7129-34.

48. Mo LP, Chen HL. One-pot, three-component condensation of aldehydes, 2-naphthol and 1,3-dicarbonyl compounds. J Chin Chem Soc 2010,57:157-61.

49. Nazeruddin GM, Pandharpatte MS, Mulane KB. Heterogeneous catalyst: Silica sulphuric acid catalysed synthesis of 9, 10-dihydro-12 aryl-8H-benzo $\alpha]$ xanthene-11 $(12 \mathrm{H})$-one derivatives under solvent free conditions. Indian J Chem 2011;50B:1532-7.

50. Khurana JM, Lumb A, Pandey A, Mangoo D. Green approaches for the synthesis of 12-aryl-8,9,10,12-tetrahydrobenzo[a]xanthen-11-ones in aqueous media and under microwave irradiation in solventless conditions. Synthesis 2012;42:1796-803.

51. Nazeruddin GM, Pandharpatte MS. Microwave promoted perchloric acid catalyzed one pot synthesis of xanthene derivatives under solventfree conditions. Der Pharm Chem 2011;3:65-71. 
52. Das B, Laxminarayan K, Krishnaiah M, Srinivas Y. An efficient and convenient protocol for the synthesis of novel 12-aryl- or 12-alkyl$8,9,10,12$-tetrahydrobenzo[a]xanthen-11-one derivatives[1]. Synthesis 2007:20:3107-12

53. Li J, Tang W, Lu L, Su W. Strontium triflate catalyzed one-pot condensation of $\beta$-naphthol, aldehydes and cyclic 1,3-dicarbonyl compounds. Tetra Lett 2008;49:7117-20.

54. Shitole NV, Sapkal SB, Shingate BB, Shingare MS. A simple and green synthesis of tetrahydrobenzo[ $\alpha]$-xanthen-11-one using peg-400 as efficient and recyclable reaction media. Bull Korean Chem Soc 2011;32:35-6

55. Nazeruddin GM, Al-Kadasi AM. Ultrasound assisted one-pot synthesis of 12-aryl -8, 9, 10, 12-tetrahydrobenzo[a]xanthen-11-one derivatives using chlorosulphonic acid as a catalyst under solvent-free conditions. Res J Pharm Biol Chem Sci 2011;2:71-6.

56. Gao S, Tsai CH, Yao CF. A simple and green approach for the synthesis of tetrahydrobenzo[a]-xanthen-11-one derivatives using tetrabutyl ammonium fluoride in water. Synlett 2009;6:949-54.

57. Kumar A, Sharma S, Maurya RA, Sarkar J. Diversity oriented synthesis of benzoxanthene and benzochromene libraries via one-pot, threecomponent reactions and their anti-proliferative activity. J Comb Chem 2010;12:20-4

58. Li JJ, Lu LM, Su WK. Anew strategy for the synthesis of benzoxanthenes catalyzed by proline triflate in water. Tetrahedron Lett 2010;51:2434-7.

59. Yimdjo MC, Azebaze AG, Nkengfack AE, Meyer AM, Bodo B, Fomum ZT, et al. Antimicrobial and cytotoxic agents from Calophyllum inophyllum. Phytochemistry 2004;65:2789-95.

60. Shoer MA, Suwanboriux K, Hobih AA, Chang CJ, Cassady JM. Xanthones and vismiones from Psorospermum febrifugum. Phytochemistry 1993;34:1413-20.

61. Miura T, Ichiki H, Iwamoto N, Kato M, Kubo M, Sasaki H, et al. Antidiabetic activity of the rhizoma of Anemarrhena asphodeloides and active components, mangiferin and its glucoside. Biol Pharm Bull 2001;24:1009-11.

62. Lin CN, Liou SS, Ko FN, Teng CM. Gamma-pyrone compounds. II: Synthesis and antiplatelet effects of tetraoxygenated xanthones. J Pharm Sci 1992;81:1109-12.

63. Guha S, Ghosal S, Chattopadhyay U.Antitumor, immunomodulatory and anti-HIV effect of mangiferin, a naturally occurring glucosylxanthone. Chemotherapy 1996;42:443-51.

64. Chen SX, Wan M, Loh BN. Active constituents against HIV-1 protease from Garcinia mangostana. Planta Med 1996;62:381-2.

65. Selvi AT, Joseph GS, Jayaprakasha GK. Inhibition of growth and aflatoxin production in Aspergillus flavus by Garcinia indica extract and its antioxidant activity. Food Microbiol 2003;20:455-60.

66. Pinto MM, Sousa ME, Nascimento MS. Xanthone derivatives: New insights in biological activities. Curr Med Chem 2005;12:2517-38.

67. Tona L, Cimanga RK, Mesia K, Musuamba CT, Bruyne TD, Apers S, et al. In vitro antiplasmodial activity of extracts and fractions from seven medicinal plants used in the democratic republic of Congo. $\mathrm{J}$ Ethnopharm 2004;93:27-32.

68. Mackeen MM, Ali AM, Lajis NH, Kawazu K, Hassan Z, Amran M, et al. Antimicrobial, antioxidant, antitumour-promoting and cytotoxic activities of different plant part extracts of Garcinia atroviridis Griff. Ex T. anders. J Ethnopharmacol 2000;72:395-402.

69. Abdel-Lateff A, Klemke C, König GM, Wright AD. Two new xanthone derivatives from the algicolous marine fungus Wardomyces anomalus. J Nat Prod 2003;66:706-8.

70. Marston A, Hamburger M, Diserens IS, Msouth JD, Hostettmann K. Xanthones from Polygala nyikensis. Phytochemestry 1993;33:809-12.

71. Chowhan ZT, Amaro AA. Pulmonary absorption studies utilizing in situ rat lung model: Designing dosage regimen for bronchial delivery of new drug entities. J Pharm Sci 1976;65:1669-72.

72. Leiro J, García D, Arranz JA, Delgado R, Sanmartín ML, Orallo F, et al. An anacardiaceae preparation reduces the expression of inflammationrelated genes in murine macrophages. Int Immunopharmacol 2004;4:991-1003.

73. a) Lin CN, Chung MI, Liou SJ, Lee TH, Wang JP. Synthesis and antiinflammatory effects of xanthone derivatives. J Pharm Pharmacol 1996;48:532-8. b) Menon S, Agarwal H, Kumar SR, Kumar SV. Green synthesis of silver nanoparticles using medicinal plant Acalypha indica Leaf extracts and its application as an antioxidant and antimicrobial agent against foodborne pathogens. Int J Appl Pharm 2017;9:42-50.

74. Garrido G, González D, Delporte C, Backhouse N, Quintero G, Núñez-Sellés AJ, et al. Analgesic and anti-inflammatory effects of Mangifera indica L. Extract (Vimang). Phytother Res 2001;15:18-21.

75. Pedro M, Cerqueira F, Sousa ME, Nascimento MS, Pinto M. Xanthones as inhibitors of growth of human cancer cell lines and their effects on the proliferation of human lymphocytes in vitro. Bioorg Med Chem 2002;10:3725-30.

76. Kolokythas G, Kostakis IK, Pouli N, Marakos P, Skaltsounis AL, Pratsinis H. Design and synthesis of some new pyranoxanthenone aminoderivatives with cytotoxic activity. Bioorg Med Chem Lett 2002; 12:1443-6.

77. Rewcastle GW, Atwell GJ, Li ZA, Baguley BC, Denny WA. Potential antitumor agents 61 . Structure-activity relationships for in vivo colon 38 activity among disubstituted 9-oxo-9H-xanthene-4-acetic acids. J Med Chem 1991;34:217-22. 